\title{
Operando Imaging of All-Electric Spin Texture Manipulation in Ferroelectric and Multiferroic Rashba Semiconductors
}

\author{
J. Krempaský, ${ }^{1}$ S. Muff, ${ }^{1,2}$ J. Minár, ${ }^{3}$ N. Pilet, ${ }^{1}$ M. Fanciulli, ${ }^{1,2}$ A. P. Weber, ${ }^{1,2}$ E. B. Guedes,${ }^{1,2}$ M. Caputo, ${ }^{1,2}$ \\ E. Müller, ${ }^{4}$ V. V. Volobuev ${ }^{5,6}$ M. Gmitra, ${ }^{7,8}$ C. A. F. Vaz, ${ }^{1}$ V. Scagnoli,${ }^{9,10}$ G. Springholz, ${ }^{5}$ and J. H. Dil ${ }^{1,2}$ \\ ${ }^{1}$ Photon Science Division, Paul Scherrer Institut, 5232 Villigen PSI, Switzerland \\ ${ }^{2}$ Institute of Physics, École Polytechnique Fédérale de Lausanne, 1015 Lausanne, Switzerland \\ ${ }^{3}$ University of West Bohemia New Technologies-Research Centre, \\ Univerzitní 8, 30614 Plzeň, Czech Republic \\ ${ }^{4}$ Electron Microscopy Facility, Paul Scherrer Institut, 5232 Villigen PSI, Switzerland \\ ${ }^{5}$ Institut für Halbleiter-und Festkörperphysik, Johannes Kepler Universität, A-4040 Linz, Austria \\ ${ }^{6}$ Institute of Physics, Polish Academy of Sciences, Aleja Lotnikow 32/46, 02-668 Warsaw, Poland \\ ${ }^{7}$ Institute for Theoretical Physics, University of Regensburg, 93040 Regensburg, Germany \\ ${ }^{8}$ Insitute of Physics, P. J. Šafárik University, 04180 Košice, Slovak Republic \\ ${ }^{9}$ Laboratory for Multiscale Materials Experiments, Paul Scherrer Institute, 5232 Villigen PSI, Switzerland \\ ${ }^{10}$ Laboratory for Mesoscopic Systems, Department of Materials, ETH Zurich, 8093 Zurich, Switzerland
}

(Received 9 July 2017; revised manuscript received 8 March 2018; published 18 June 2018)

\begin{abstract}
The control of the electron spin by external means is a key issue for spintronic devices. Using spin- and angle-resolved photoemission spectroscopy (SARPES) with three-dimensional spin detection, we demonstrate operando electrostatic spin manipulation in ferroelectric $\alpha$-GeTe and multiferroic $\mathrm{Ge}_{1-x} \mathrm{Mn}_{x} \mathrm{Te}$. We demonstrate for the first time electrostatic spin manipulation in Rashba semiconductors due to ferroelectric polarization reversal. Additionally, we are also able to follow the switching pathway in detail. In multiferroic $\mathrm{Ge}_{1-x} \mathrm{Mn}_{x}$ Te operando SARPES reveals switching of the perpendicular spin component due to electric-field-induced magnetization reversal. This provides firm evidence of magnetoelectric coupling which opens up functionality with a multitude of spin-switching paths in which the magnetic and electric order parameters are coupled through ferroelastic relaxation paths. This work thus provides a new type of magnetoelectric switching intertwined with Rashba-Zeeman splitting in a multiferroic system.
\end{abstract}

DOI: 10.1103/PhysRevX.8.021067

\section{INTRODUCTION}

Manipulating the spin texture of ferroelectrics (FE) through electric fields and of multiferroics through both magnetic and/or electric fields is a key requirement for programmable spintronic devices [1]. The recent discovery of a giant bulk Rashba-type spin splitting in ferroelectric $\alpha$-GeTe adds a promising candidate for such devices and raises the question of whether the spin texture in such a material can be modulated by electric fields by controlling the inversion asymmetry and thus also the Rashba splitting [2,3]. Such devices would strongly benefit from the large bulk Rashba spin splitting of $\alpha$-GeTe even at room temperature [4]. This spin splitting is exemplified in Fig. 1(a), which shows the $\alpha$-GeTe bands near the $Z$ point

Published by the American Physical Society under the terms of the Creative Commons Attribution 4.0 International license. Further distribution of this work must maintain attribution to the author(s) and the published article's title, journal citation, and DOI.
Subject Areas: Condensed Matter Physics,

Materials Science, Spintronics of the Brillouin zone where the Rashba splitting is most pronounced [5-7]. Ideally, electric-field-induced ferroelectric switching will change the spin orientation of these bands as sketched in Fig. 1(b), thus allowing a reprogramming of spin currents by external means.

$\alpha$-GeTe is the simplest known binary ferroelectric semiconductor with a narrow band gap [4,8]. Below $T_{C} \approx$ $700 \mathrm{~K}$ it assumes a noncentrosymmetric rhombohedral structure in which an electric dipole is formed due to a relative $\mathrm{Ge} / \mathrm{Te}$ sublattice displacement along the [111] direction [see Fig. 1(c)]. For moderate Mn dopings, $\mathrm{Ge}_{1-x} \mathrm{Mn}_{x} \mathrm{Te}$ becomes ferromagnetic as well $[9,10]$, which opens up new spin-based functionalities because this multiferroicity entangles the Rashba and Zeeman effects within a three-dimensional system [5,11]. In this new class of multiferroic Rashba semiconductors (MUFERS), we already showed that external magnetic fields switch the bulk spin texture [5]. In this work, we demonstrate that spin manipulation is also possible by electric fields, meaning that the magnetoelectric coupling so far induced only by magnetic fields [5,9] expands even to all-electric control 
(a)
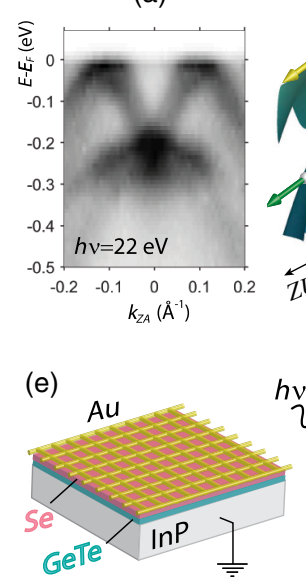

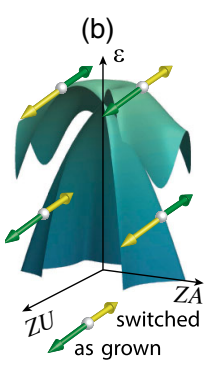

(f)

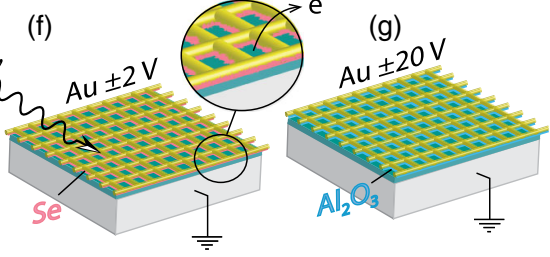

FIG. 1. (a) ARPES band map of $\alpha$-GeTe(111) along $A-Z-A$. (b) Schematic representation of the two highest occupied Rashbasplit valence bands around the $Z$ point of the Brillouin zone (as-grown spin winding in green, switched in yellow). (c) $\alpha$-GeTe unit cell with FE displacement of the Ge atoms along [111] (green arrow). (d) Ferroelectric switching of $\alpha$-GeTe into the [111]

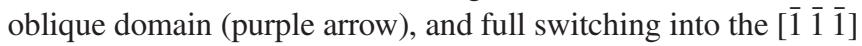
direction (yellow arrow). Schematic layout of the gated sample used for operando SARPES before (e) and after (f) annealing. (g) Improved design with $\mathrm{Al}_{2} \mathrm{O}_{3}$ dielectric coating on the gold grid.

of magnetism. Thus, from an application viewpoint, $\mathrm{Ge}_{1-x} \mathrm{Mn}_{x} \mathrm{Te}$ fulfills all criteria for mutual control of magnetism and ferroelectricity via magnetoelectric coupling effects [12,13].

Our epitaxial films, grown by molecular beam epitaxy [9] on conducting $\operatorname{InP}(111)$ substrates, were studied by means of spin- and angle-resolved photoemission under applied voltage (operando SARPES) at $20 \mathrm{~K}$, and complemented by piezoresponse force microscopy (PFM) investigations (see Appendix A and Refs. [4,6,14,15]). To understand the functional electronic properties of $\alpha$-GeTe, we need to consider its FE lattice structure in which the Ge atoms are displaced along [111] with respect to the six neighboring Te atoms as sketched in Fig. 1(c). The displacement, indicated by the green arrow, is very large, reaching almost $10 \%$ of the lattice constant $[4,10]$. An important issue for switching of the polarization is that, due to the fourfold degeneracy of the rhombohedral lattice distortion, eight different domains with individual polarization vectors pointing along different $\langle 111\rangle$ directions may be present. This multidomain structure is independent of the substrate epitaxial registry because thin films grown on $\mathrm{BaF}_{2}$ or $\mathrm{Si}(111)$ develop the same major and secondary FE domains $[4,9,10,16]$. In such a multidomain structure, polarization reversal may involve intermediate steps via oblique [111] domains [purple arrow in Fig. 1(d)], typically leading to a whole realm of FE-fatigue effects [17].

\section{EXPERIMENTAL METHODS AND SETUPS}

SARPES allows one to directly determine the spin texture of the electronic band structure $[18,19]$ and therefore probe in operando the reversal of the Rashba spin polarization as a function of the applied electric field. The layout of the novel gated sample structure developed for such measurements is shown in Fig. 1(e) and consists of a protective Se cap on top of the $\alpha$-GeTe or $\mathrm{Ge}_{1-x} \mathrm{Mn}_{x} \mathrm{Te}$ epilayers onto which a $\mathrm{Au}$ mesh is placed as a top electrode. The bottom electrode is formed by the conductive InP substrate, which is grounded through the sample holder. Two strategies were employed in the device assembly. In the first [Fig. 1(f)], the in situ desorbed Se cap sticks to the bottom side of the $\mathrm{Au}$ mesh providing a quasi-insulating contact between the $\mathrm{Au}$ gate and the semiconducting $\alpha$-GeTe. The second assembly [Fig. 1(g)] employs an $\mathrm{Al}_{2} \mathrm{O}_{3}$ layer predeposited on the $\mathrm{Au}$ mesh to provide better dielectric insulation. Consequently, higher bias voltages can be applied and the setup can withstand several annealing treatments to rejuvenate the sample after electric cycling. Both setups have similar capacitorlike structures and yield consistent results in the sense that, as shown below, when applying first a negative voltage on the top gate, consistent spin rotations are obtained. Further technical details of the sample environment which enable the operando SARPES are discussed in Appendix A. To verify that the observed spin rotation is not an artifact of the setup, we carry out a similar measurement on the wellcharacterized $\mathrm{Bi}_{2} \mathrm{Se}_{3}$ topological insulator [20,21]. As the gate control does not affect the measured spin polarization of the $\mathrm{Bi}_{2} \mathrm{Se}_{3}$ surface state, the $\alpha$-GeTe spin texture manipulation discussed below is indeed related to ferroelectric control of the Rashba bands.

\section{ELECTRIC CONTROL OF SPIN ORIENTATION}

The bulk $\alpha$-GeTe bands are shadowed by surface resonances (SR) which in ARPES typically display higher spectral weight compared to bulk states [11]. Moreover, they disperse with photon energy [4,7] and are thus easily confused with bulk states [6]. Therefore, in ARPES one observes metallic states at $E_{F}$, in general agreement with the intrinsic $p$-type doping from Ge vacancies thought to be responsible for the metallic character of the nominally semiconducting GeTe [6,22].

Figures 2(a) and 2(b) show ARPES spectra measured from the first sample setup with an applied bias voltage of -1.6 and $+1.6 \mathrm{~V}$, respectively; the experimental geometry is indicated in Fig. 2(d). The data clearly show that part of the applied voltage falls off between the $\mathrm{Au}$ mesh and the GeTe film $(\approx 0.2 \mathrm{~V})$ and the rest $(\approx 1.4 \mathrm{~V})$ over the 200-nm-thick film. The shift of the Fermi level with applied voltage and its reversal as a function of polarity is evident from Fig. 10(a) in Appendix A. As a whole the gated 
(a)

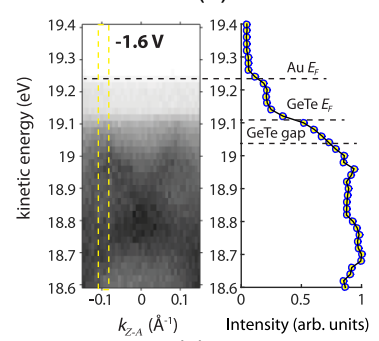

(C)

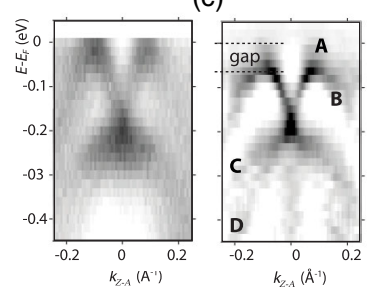

(e)

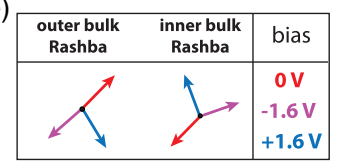

(b)

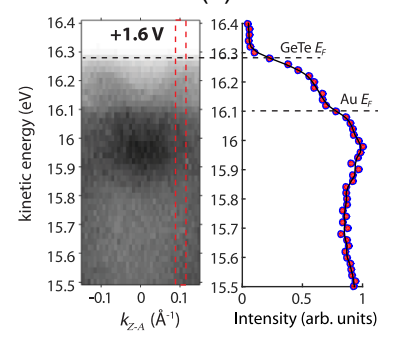

(d)

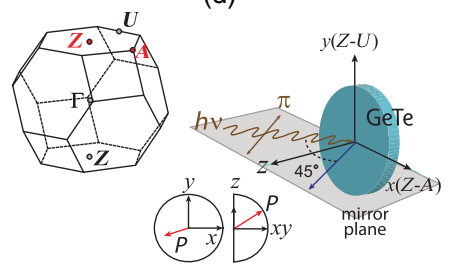

(f)

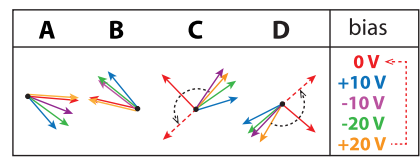

FIG. 2. (a) Raw ARPES band map of $\alpha$-GeTe from the first gated sample [Fig. 1(f)], measured along the $Z-A$ direction for negative $(-1.6 \mathrm{~V})$ and (b) positive applied voltage $(+1.6 \mathrm{~V})$. Right: EDC cuts integrated over the dashed rectangles. Indicated are two Fermi levels from the $\alpha$-GeTe and the Au gate. (c) (left) Raw ARPES band map from the improved gated sample [Fig. 1(g)] and (right) curvature fitting [23] to better visualize the bands. (d) $\alpha$-GeTe Brillouin zone (BZ) and experimental geometry with definition of the local $x y z$ axes and polar representation of the 3D spin vectors. (e),(f) Bias-dependent in-plane $P_{x, y}$ vectorial spin analysis of the bands indicated in (c). The bias control sequence is color coded. Dashed arrows in (f) indicate the $+20 \rightarrow 0 \mathrm{~V}$ spin relaxation transition.

sample, including contact between GeTe and the Au mesh, shows an $I(V)$ curve with a rectifying behavior [see Fig. 10(b) in Appendix A]. Despite the fact that there is a semiconducting current leakage, we can rule out significant Joule heating and eventual changes in the chemical composition during the poling cycles [see Fig. 10(c) in Appendix A]. The field effect in the gated sample is evident and reproducible also for the improved setup shown in Fig. 1(g) without any current leakage.

In agreement with our earlier studies on bare $\alpha$-GeTe near the $Z$ point $(h \nu=22 \mathrm{eV})$, where the bulk Rashba splitting is most pronounced, we discern the valence band maximum beneath a narrow gap $(\approx 70 \pm 20 \mathrm{meV})$ [4]. Compared to Fig. 1(a), the quality of the spectra in Figs. 2(a) and 2(b) is affected by the top Au mesh and the remains of the Se cap, yet the data from the second gated sample in Fig. 2(c) are clear enough to locate the bulk bands shadowed by SR bands in accordance with previous studies $[4,5,11]$.

Next we focus on the effect of the electric field on the Rashba-split states in spin-resolved measurements. For this

purpose, we visualize the SARPES data as spin-resolved momentum distribution curves (MDC) and energy distribution curves (EDC) recorded at selected bias voltages. For clarity, Figs. 2(e) and 2(f) summarize the in-plane operando modulation of the spin orientations. In agreement with SARPES data from $\alpha-\mathrm{GeTe} / \mathrm{BaF}_{2}(111)$, the measured pristine spin texture shows a canted spin arrangement different from the ideal Rashba model [4,24].

The SARPES data and the vectorial spin fitting from the two sample designs are detailed in Figs. 3 and 4, respectively. These figures directly demonstrate the operando spin manipulation. The upper panels $[(a)-(c)]$ show the SARPES total intensity and peak fits of the indicated bands; their 3D spin polarizations and spin currents are shown in the lower panels $\left[\left(\mathrm{a}^{\prime}-\mathrm{c}^{\prime}\right)\right]$. In Figs. 3(d) and 4(d), the band model is depicted together with the positions where the cuts were taken. We emphasize that the measured spin polarizations clearly change with different applied voltages, independently of the spin fitting details.

Based on our combined experimental and theoretical study over a wide photon energy range, we were able to locate a region in momentum space where we are primarily sensitive to the Rashba-split bulk states and not to the surface resonance states [4]. This range is covered in the measurement in Fig. 3 and can therefore be considered as representative of the bulk spin orientation. As summarized in Fig. 2(e), both the inner and the outer Rashba states show an almost perfect spin reversal in the $0 \rightarrow-1.6 \mathrm{~V}$ transition, and an incomplete in-plane spin rotation in the $-1.6 \rightarrow+1.6 \mathrm{~V}$ transition. This clearly demonstrates that the spin texture can be manipulated by the applied voltage.

Similar electric spin manipulation is observed in the sample with improved dielectric coating on the top gate and operating at higher bias voltages. The EDC results are summarized in Fig. 2(f) and detailed in Fig. 4. Already in the raw spin polarization data it is clear that all three spin components are significantly changed for the measurement with $+10 \mathrm{~V}$ compared to the unbiased sample $(0 \mathrm{~V})$. For a more systematic analysis and in order to summarize all measurements in vector plots, we have fitted the data with 4 peaks (A-D) to account for the bulk states and the surface resonances. However, one has to be careful to make a rigid connection between the peaks and their bulk or surface resonance character because the states are strongly hybridized and their spin direction is influenced by interband spin-orbit coupling [25]. Based on our previous photoemission studies [4,11], a simplified $\alpha$-GeTe band model relevant for our operando SARPES studies, denoted in Figs. 3(d) and 4(d), consists of surface resonances indicated by black dashed lines and bulk Rashba bands denoted with red and blue solid lines.

In order to systematically test the magnitude of the spin rotation with poling polarity, in Fig. 4 we first apply a positive voltage $(+10 \mathrm{~V})$ and find that the magnitude of the spin rotation is smaller compared to the results from Fig. 3. 


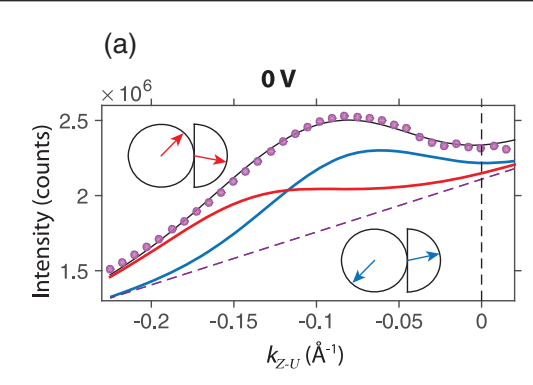

$\left(a^{\prime}\right)$

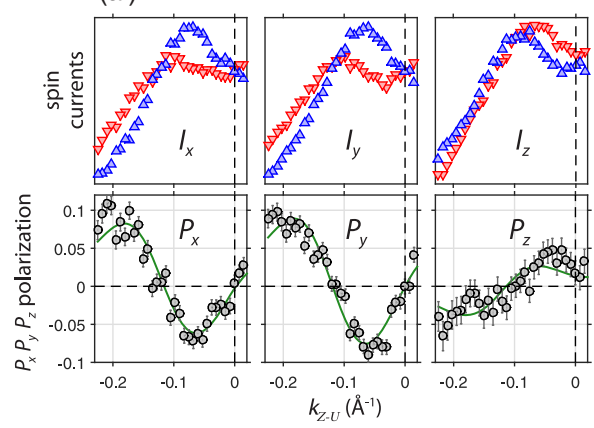

(b)

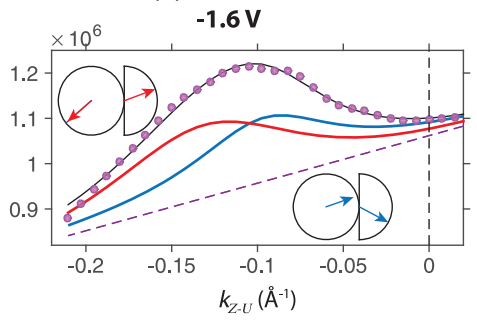

$\left(\mathrm{b}^{\prime}\right)$

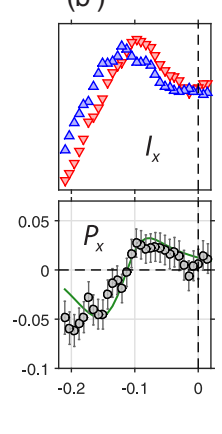

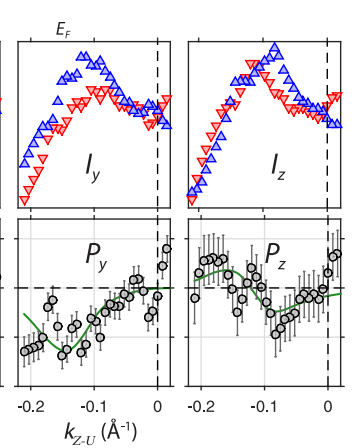

(c)

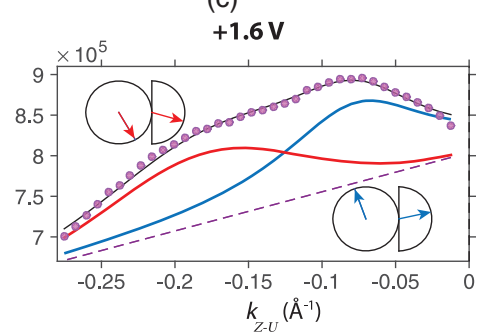

(d) $E(\mathrm{eV}) \uparrow$

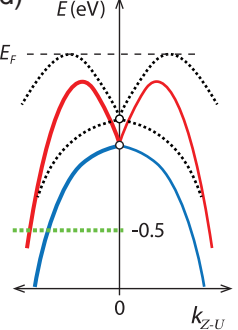

$\left(c^{\prime}\right)$

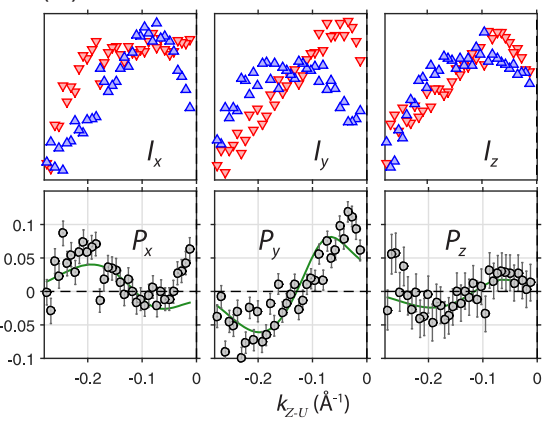

FIG. 3. Three-dimensional bias-dependent vectorial spin analysis from the first poling device sketched in Fig. 1(f). (a) As-grown sample, (b) $-1.6 \mathrm{~V}$, and (c) $+1.6 \mathrm{~V}$ bias voltage. SARPES total intensity is indicated in purple dots, fits of the Rashba-split bulk bands are depicted as red and blue lines, the arrows represent 3D spin expectation values, their $P_{x, y}$ projections are also summarized in Fig. 2(e). $\left(\mathrm{a}^{\prime}\right)-\left(\mathrm{c}^{\prime}\right)$ Spin currents $I_{x, y, z}$ and $P_{x, y, z}$ polarizations including data fits (green lines). Data are obtained along the MDC path indicated by the green dashed line in (d) at a binding energy of $0.5 \mathrm{eV}$.

Furthermore, the spin rotation appears to not fully follow the changes of applied electric field after the first poling cycle, as is already directly clear from the $-10 \mathrm{~V}$ data. This means that electric spin manipulation becomes blocked after the second cycling, an effect referred to as fatigue.
Surprisingly, besides stalled electrostatic spin reorientation, our SARPES data also reveal unexpected spin switching when the system relaxes from a relatively high bias voltage, reminiscent of ferroelectric backswitching dynamics activated by charge agglomerates at the domain walls (a)

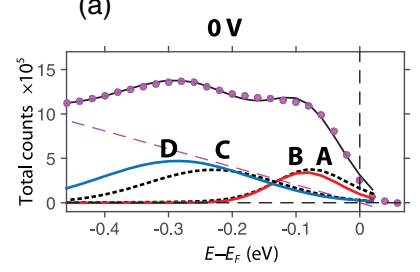

$\left(a^{\prime}\right)$

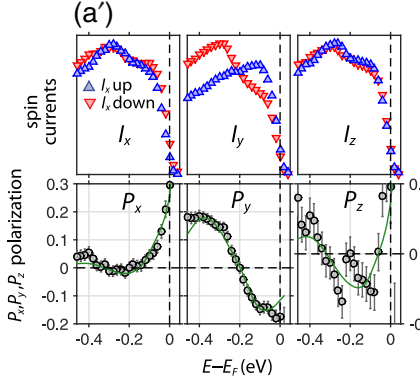

(b)

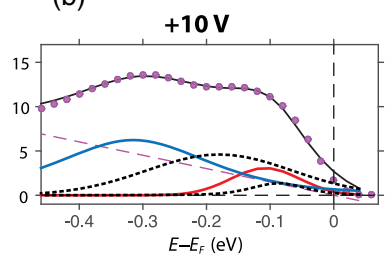

$\left(b^{\prime}\right)$ (c)

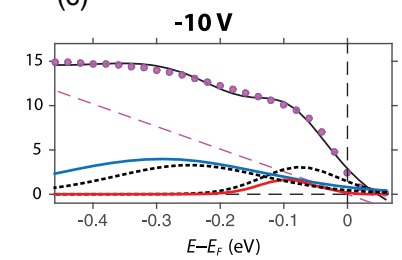

$\left(c^{\prime}\right)$

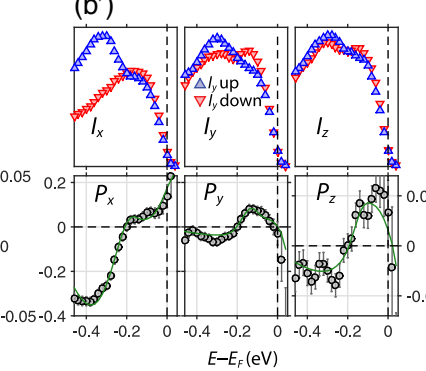

(d)

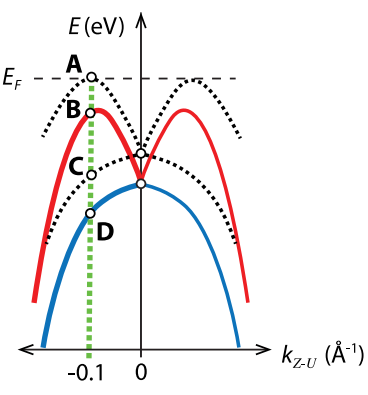

(e)

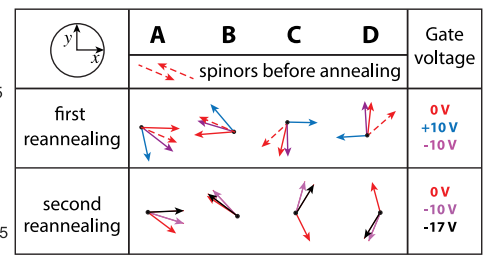

FIG. 4. Three-dimensional bias-dependent vectorial spin analysis from the second poling device sketched in Fig. 1(g). (a) As-grown sample, (b) $+10 \mathrm{~V}$, and (c) $-10 \mathrm{~V}$ bias voltage. SARPES total intensity (purple dots) and peak fitting of the bands A-D. (a') $-\left(\mathrm{c}^{\prime}\right)$ Spin currents $I_{x, y, z}$ and $P_{x, y, z}$ polarizations including their fits (green lines). Data are obtained along the EDC path indicated by a green dashed line in (d) at $k_{\|}=-0.1 \AA^{-1}$. (e) 3D spin vectors for each of the bands obtained after ferroelectric rejuvenation with annealing. For comparison, dashed red arrows indicate the ferroelastic relaxation transition from Fig. 2(f) before the rejuvenation. 
[26]. We find that this is exactly the case for the sudden, almost $180^{\circ}$ spin rotation in the $+20 \rightarrow 0 \mathrm{~V}$ relaxation transition denoted by dashed arrows in Fig. 2(f), thus indicating that ferroelastic effects also have to be considered in our operando SARPES measurements. We note that the observed ferroelastic effects are probably related to the (metavalent) resonant-bonding mechanism of $\mathrm{GeTe}$, which leads to a decrease of the relative stability of the FE order [27]. However, as discussed below, the critical aspect that determines the ferroelastic effects is likely due to formation of isolated ferroelastic domains, which influence the ferroelectric switching dynamics.

\section{FE DOMAINS PROBED BY PFM AND OPERANDO SARPES}

To elucidate the polarization reversal induced by the applied electric field and the reduced spin rotation after the initial poling cycle, PFM studies were performed on the samples as shown in Figs. 5(a)-5(f). In this technique,

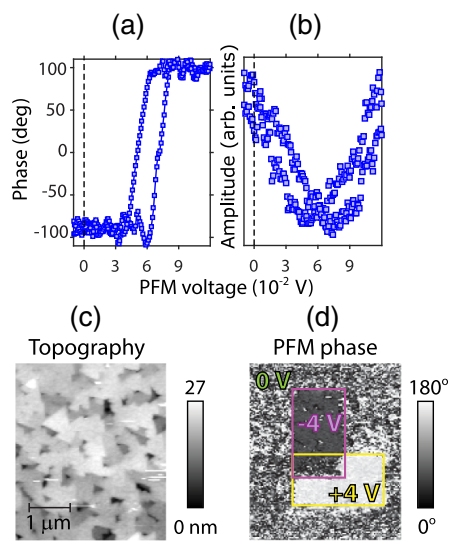

(e)
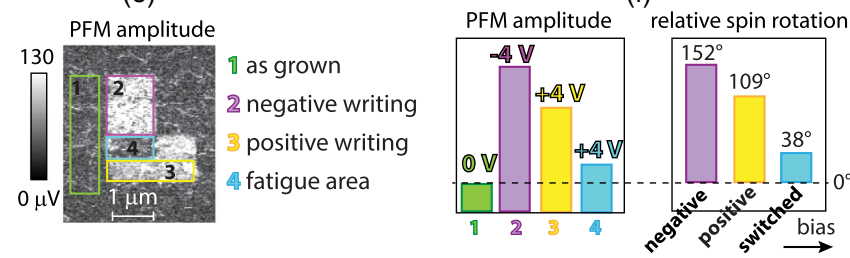

FIG. 5. (a),(b) PFM vertical phase and amplitude hysteresis loop measured on the $\alpha$-GeTe/InP(111) surface in spectroscopy mode. (c) Surface topography measured in atomic force microscopy mode. (d) PFM phase in domain mapping of two intersecting areas with $\pm 4 \mathrm{~V}$. (e) Associated PFM amplitude signal. (f) (left) Average amplitude signal from selected areas: (1) pristine area in green, (2) $-4 \mathrm{~V}$ written domain in magenta, (3) $+4 \mathrm{~V}$ written domain in yellow, (4) intersection area of (2) and (3) in blue, indicating the fatigue area. (Right) corresponding SARPES average spin rotation magnitudes: applying first negative bias (magenta), first positive bias (yellow), and switched bias voltage (blue). (g) As-grown unit cell of surface relaxed $\alpha$-GeTe with outof-plane FE polarization (green arrow). (h) Surface relaxation after switching the $\alpha$-GeTe bulk atoms in the dashed rectangle indicated in $(\mathrm{g})$. excitation of the sample via an ac voltage applied between sample and probe tip induces an oscillation of the cantilever deflection through the inverse piezoelectric effect. Its amplitude is a measure for the absolute magnitude of the piezoresponse, and its phase corresponds to the polarization direction. Figure 5(a) shows the hysteresis loop of the PFM phase as a function of dc tip voltage, evidencing the reversal of the polarization direction in the $\alpha$-GeTe films induced by the electric field, which corresponds exactly to the spin reversal that we observe by SARPES. We emphasize that the shift of the hysteresis loop, similar to that in Ref. [6], is also observed in the characteristic "butterfly" shape of the amplitude signal in Fig. 5(b) [14], thus suggesting a preferential built-in polarization direction related to the surface termination. Such experimental observation calls for a more accurate investigation of the PFM data, as well as theoretical models reflecting the FE-domain switching in the $\alpha$-GeTe surface region.

In addition to the local piezoelectric response in Figs. 5(a) and 5(b), a nonvolatile electric writing of FE domains of $\alpha$-GeTe by applied dc voltages is demonstrated by $180^{\circ}$ phase reversal in Fig. 5(d). The associated amplitude image of oppositely poled regions written in the sequence $1-3$ from $0 \rightarrow-4 \rightarrow+4 \mathrm{~V}$ is indicated by the green, magenta, and yellow boxes in Fig. 5(e), respectively. We note that the PFM phase domain mapping remains unchanged even after 2 days, thus ruling out charging artifacts induced by PFM in the FE switching. In order to correlate the magnitude of the electrostatic spin manipulation with our PFM data, the left-hand panel in Fig. 5(f) shows the average PFM amplitude signal from four areas: pristine (green), negative (magenta), and positive (yellow) domain writing, and the twice-written area 4 indicated in blue [the areas are indicated in Fig. 5(e)]. Because the PFM amplitude signal is proportional to the projection of the $\mathrm{FE}$ polarization perpendicular to the sample surface, data in the left-hand panel of Fig. 5(f) show that it is easier to write domains with negative than with positive voltages.

For a quantitative comparison between PFM and SARPES, Fig. 5(f) shows the average magnitude of the relative spin rotations with respect to the as-grown or rejuvenated samples, after applying first negative bias (magenta), first positive bias (yellow), and finally for switched bias voltage (blue). This comparison indicates that, consistently with the PFM amplitude signal, first application of a negative voltage results in a larger change of spin polarization angle compared to the pristine case than first application of positive voltage. This suggests that direct piezoresponse in PFM and indirect spin manipulation via Rashba-type coupling to FE order give consistent quantitative results. Furthermore, the partial spin reorientation after the second switching is in accordance with the significantly reduced amplitude signal inside the overwritten area 4, indicated in blue in Fig. 5(e). These results 
indicate a rapid degradation in the ferroelectric response not expected in ideal ferroelectrics.

In fact, a more detailed structural analysis with transmission electron microscopy (TEM) (Fig. 9 in Appendix A) reveals that the characteristic $\alpha$-GeTe surface topography in Fig. 5(c) originating from stacking faults [28] extends into a lamellar structure in the entire film by forming at least two types of grains defining isolated crystalline regions. This readily indicates that the system is far from forming the ideal monodomain structure needed for ideal spinswitching dynamics. The related ferroelectric order with many grain boundaries is the primary candidate for carrier agglomeration, also reflected in the $\alpha$-GeTe intrinsic $p$-type transport properties [22]. Under such conditions it is reasonable to assume that the partial spin reorientation after the second switching originates from the existence of depolarization fields due to unipolar $\mathrm{FE}$ fatigue near domains and/or grain boundaries [29,30].

Because of the small escape depth of photoelectrons, SARPES is a surface sensitive technique and therefore the role of the film surface must be taken into account. To this end, we performed $a b$ initio calculations based on density functional theory (see Appendix C) to assess the equilibrium surface structure as a function of applied electric field. In the calculations, the bulk FE switching was implemented by switching the inner electric field in the bulk region [dashed rectangle in Fig. 5(g)], which results in a substantial structural rearrangement of the surface region as shown by Fig. 5(h). Given that the Te-terminated surface is preferred for $\alpha$-GeTe [4,6,31], the strong electronegativity of the surface Te atoms imposes an unipolar FE polarization at the surface.

The surface relaxation associated with FE switching induces a subsurface FE domain wall (similar to the rocksalt $\beta$-GeTe crystal structure), underneath of which the bulk $\alpha$-GeTe FE order is reversed [yellow area in Fig. 5(h)]. This triple-layer domain wall separating oppositely oriented domains is an essential ingredient in the FE-switching mechanism, which involves swapping between the longer and shorter Ge-Te bonds [14]. Near the surface the formation of the domain wall gives rise to a depolarization field $p_{z}{ }^{\prime}$, which we associate with asymmetric hysteresis loops such as seen in Fig. 5(a). We note that charge agglomeration is expected to occur in the subsurface domain wall region and screen the bulk bands, hence hindering the full spin reversal in repetitive electric cycles in agreement with our operando SARPES results.

A way to test the contribution of unipolar FE fatigue to the switching behavior is thermal annealing below the FE Curie point [30]. Figure 4(e) summarize SARPES data taken for two consecutive sample reannealings to temperatures around $520-540 \mathrm{~K}$, well below the $T_{C}$ of $700 \mathrm{~K}$. The red dashed vectors indicate the last spin orientation after the $+20 \rightarrow 0 \mathrm{~V}$ transition discussed above before first reannealing. Consistent with the results summarized in
Figs. 2(e) and 2(f), significant electrostatic spin manipulation is obtained only in the first poling cycle right after each FE rejuvenation. We find that after FE rejuvenation the spin orientation is restored to the initial values, which is indicative of the unipolar $\mathrm{FE}$ fatigue due to charge agglomeration at $\alpha$-GeTe grain boundaries and/or domain walls $[29,30]$. This means that isolated ferroelastic domains become immobile and thus block the spin manipulation in SARPES, which is in agreement with the fatigue area observed in PFM and the domain wall analogue in the theoretical model. This experimental observation is evident in SARPES data measured from gated samples with and without improved dielectric properties summarized in Figs. 2(f) and 4(e), respectively; and is direct evidence that the electrostatic field effect responsible for spin manipulation is present in both gated samples.

\section{ELECTRIC-FIELD-INDUCED MAGNETIZATION REVERSAL IN Ge ${ }_{1-x} \mathrm{Mn}_{x} \mathrm{Te}$}

We now turn to electric spin manipulation of multiferroic $\mathrm{Ge}_{1-x} \mathrm{Mn}_{x} \mathrm{Te}$. In comparison to ferroelectrics, in MUFERS there is an additional spontaneous magnetization superimposed over the FE polarization [5]. In addition, because of the magnetoelectric coupling between magnetization and polarization, field-induced switching of polarization can also induce a switching of magnetization, thus providing additional means of spin control. A particularly interesting feature of $\mathrm{Ge}_{1-x} \mathrm{Mn}_{x} \mathrm{Te}$ is that the easy axis of magnetization $\left(M_{z}\right)$ and the electric dipole moment $\left(p_{z}\right)$ are collinear and perpendicular to the surface [5,9]. As a result, the Rashba bands are Zeeman split and thereby assume an additional out-of-plane spin polarization $P_{z}$ around the $Z$ point. As illustrated in Fig. 6(a), this out-of-plane spin component is directly linked to the magnetization direction [5]. Thus, the switching of $M_{z}$ can be directly detected by measuring $P_{z}$ in SARPES.

Figures 6(d)-6(f) present the measured $P_{z}$ of the $\mathrm{Ge}_{0.87} \mathrm{Mn}_{0.13}$ Te sample across the Zeeman gap in normal emission [green dashed line in Fig. 6(b)] under different bias conditions. Clearly, the spin polarization spectrum $P_{z}(E)$ switches sign upon bias reversal of the gate, indicating an induced reversal of the magnetization by the applied electric field. This experimental result presents the first unambiguous and direct evidence for the existence of a strong magnetoelectric coupling between the electric dipoles and the magnetic moments in this material.

Contrary to $\alpha$-GeTe, where the spin gate control becomes blocked after the first poling cycle, SARPES of $\mathrm{Ge}_{0.87} \mathrm{Mn}_{0.13} \mathrm{Te}$ is found to respond to each change of the external electric field. This is demonstrated by Figs. 6(d)-6(f) where the $P_{z}$ data, collected at each step of the color-coded electric field sequence, are presented in comparison with that recorded at $+10 \mathrm{~V}$ (blue symbols). After each switching cycle the modification in the $P_{z}(E)$ 
(a)

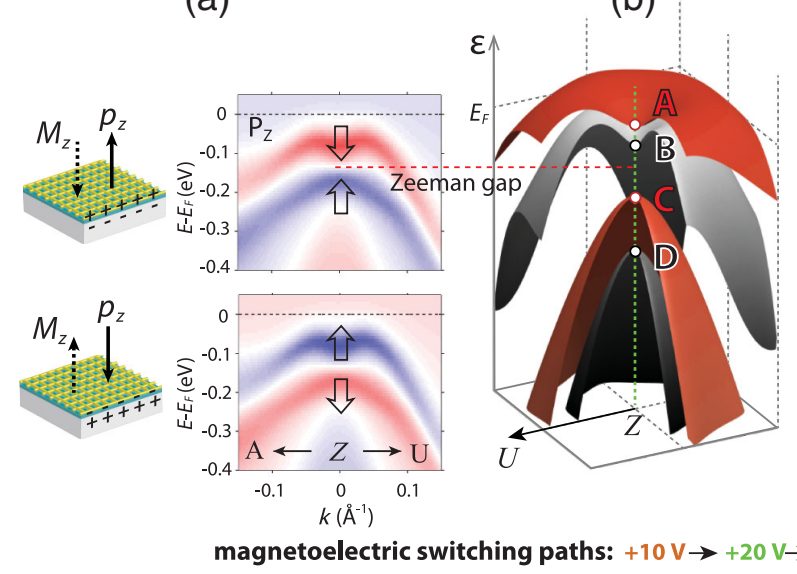

(c)

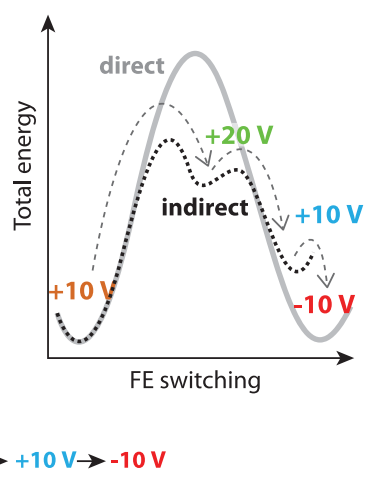

(d)

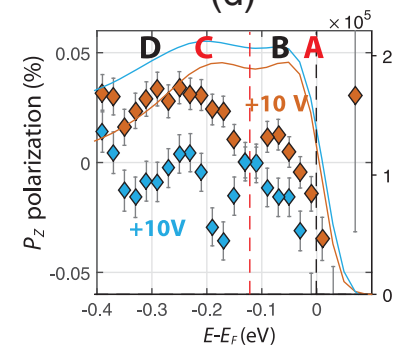

(e)

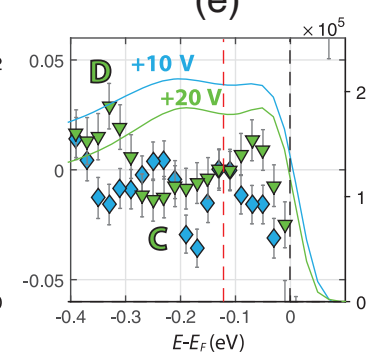

(f)

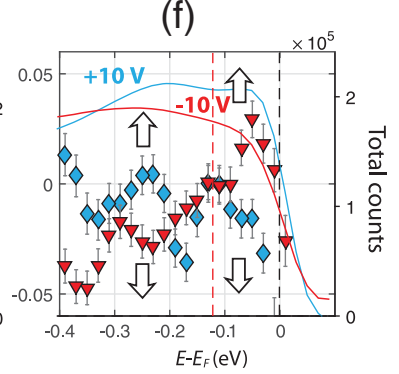

FIG. 6. (a) Calculated out-of-plane spin polarization $P_{z}$ for the bulk bands of $\mathrm{Ge}_{0.87} \mathrm{Mn}_{0.13}$ Te (from Ref. [5]): (top) as-grown configuration, (bottom) switched configuration. (b) Schematic representation of the $\mathrm{Ge}_{1-x} \mathrm{Mn}_{x}$ Te surface electronic structure with Rashba-Zeeman split bulk bands B-D and their surface resonance replica A-C. (c) Schematic diagram of direct and kinetically favored indirect multiferroic switching paths. (d)-(f) SARPES data resolved across the Zeeman gap in normal emission (left axis, symbols), overlaid with spin-integrated EDC intensities (right axis, full lines). The arrows in (f) indicate the $P_{z}(E)$ dependence switching upon bias reversal of the gate.

spectrum can be attributed to a weakening of the pinning strength of the FE polarization in $\mathrm{Ge}_{0.87} \mathrm{Mn}_{0.13}$ Te compared to $\alpha$-GeTe. This is explained by the fact that the lattice distortion and off-center displacement of the Ge atom with respect to the Te atoms in $\mathrm{Ge}_{0.87} \mathrm{Mn}_{0.13} \mathrm{Te}$ decreases with increasing Mn content $[9,10]$. This evidently reduces the energy barriers for switching of the atomic positions in the FE reorientation and thus leads to a softening of the FE properties. As illustrated in Fig. 6(c), the coupling to the magnetization can give rise to complex switching paths that will result in unconventional spin texture evolutions as a function of biasing sequence.

\section{CONCLUDING REMARKS}

In summary, we demonstrate all-electric spin manipulation in ferroelectric and multiferroic Rashba semiconductor devices using operando spin-resolved photoemission spectroscopy. The results give direct evidence for electrostatic tuning of the spin texture. Moreover, from the out-of-plane spin polarization we demonstrate magnetization reversal in multiferroic $\mathrm{Ge}_{0.87} \mathrm{Mn}_{0.13}$ Te by applying electric fields, which provides unambiguous evidence for the strong magnetoelectric coupling between the ferroelectric and magnetic order in this system. Ferroelectric fatigue and ferroelasticity are found to play a crucial role. Therefore, robust all-electric spin switching between remnant polarization states requires further improvements in domain stabilization and sample growth. In this respect we demonstrate that the PFM amplitude signal is reflected in the electrostatic spin manipulation, providing an efficient tool to study and optimize this type of material for all-electric spin manipulation. Our experimental findings open up a promising path toward robust and programmable semiconductor-based spintronics with functionality coupled to electronic and magnetic properties.

\section{ACKNOWLEDGMENTS}

The authors gratefully acknowledge constructive discussions with V. N. Strocov. This work was supported by the Swiss National Science Foundation Projects No. PP00P2 144742 and PP00P2_170591, and the Austrian Science Funds Project No. P 30960. M. G. acknowledges the German funding agency DFG SFB 689 and J. M. acknowledges the European Regional Development Fund (ERDF), project CEDAMNF, Reg. No. CZ.02.1.01/0.0/0.0/15_003/ 0000358. 


\section{APPENDIX A: EXPERIMENTAL TECHNIQUES}

Experiments were performed on 200-nm-thick ferroelectric Te-terminated $\alpha$-GeTe and (GeMn)Te films grown by molecular beam epitaxy on $\operatorname{InP}(111)$ substrates. $\alpha$-GeTe grown on $\operatorname{InP}(111)$ substrates features significantly smaller FE coercive fields compared to $\mathrm{Si}(111)$ substrates $[6,14,15]$ and is therefore an optimal choice for operando photoemission studies in our gated samples. The sample surface in Fig. 7(a) has a protective stack of amorphous Te- and Se-capping layers with a total thickness of $200 \mathrm{~nm}$ used to avoid surface degradation and oxidation. A complete or partial removal of the protective Te/Se stack in the ultrahigh vacuum chamber was achieved by annealing at temperatures around $570 \mathrm{~K}$.

SARPES experiments were performed at the COPHEE end station of the Surfaces and Interfaces Spectroscopy beam line at the Swiss Light Source (SLS) synchrotron radiation facility, Paul Scherrer Institut, Switzerland, using $p$-polarized, $h \nu=22 \mathrm{eV}$ photons, unless indicated otherwise. An Omicron EA125 hemispherical energy analyzer is equipped with two orthogonally mounted classical Mott detectors [32]. The whole setup allows one to measure simultaneously all three spatial components of the spinpolarization vector for each point of the band structure. The SARPES data were measured with the sample azimuths $Z-U$ or $Z-A$ aligned perpendicular to the scattering plane, as denoted in Fig. 2(d). The angular and combined energy resolution were $1.5^{\circ}$ and $60 \mathrm{meV}$, respectively. In spinintegrated mode these resolutions were set to $0.5^{\circ}$ and $20 \mathrm{meV}$. All SARPES data were collected at $20 \mathrm{~K}$, measured by a Si diode placed near the sample.

Figures 7(a) and 7(b) show images and technical details of the sample holder used for operando SARPES, and for the annealing needed for both sample preparation and ferroelectric rejuvenation. The electroformed $\mathrm{Au}$ mesh used in the poling device had a nominal aperture of (a)

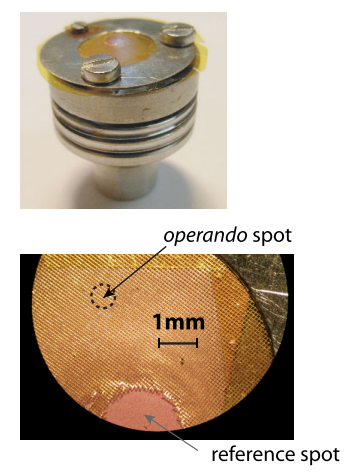

(b)

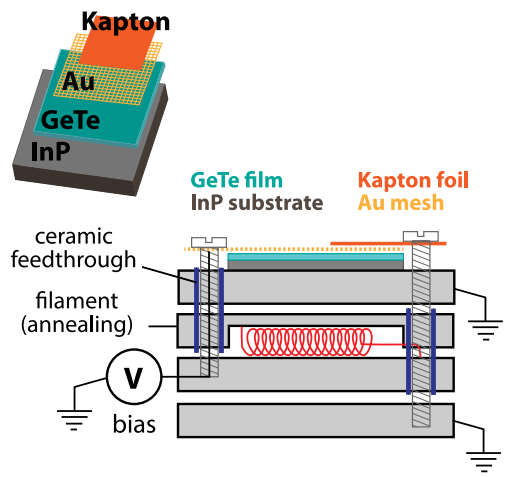

FIG. 7. (a) Optical images of the SARPES sample holder showing the Au mesh on top of the sample. (b) Schematic layout of the gated sample assembly showing the electric connections for floating bias control, sample heating, and Au-mesh isolation with Kapton foil.
$64 \mu \mathrm{m}$, thickness $4 \mu \mathrm{m}$, and $70 \%$ open area. The sample holder was adapted for floating voltage gate control of the $\mathrm{Au}$ mesh isolated from the manipulator ground. The InP substrate was grounded through the sample holder during the operando SARPES measurements and the bias voltage was applied to the Au mesh. The measured kinetic energies were adjusted according to the applied voltage. Care was taken to avoid a direct grounding of the front of the sample and the Au mesh by means of Kapton foils. For measuring reference spectra without Au mesh we used a reference spot shown in the bottom of Fig. 7(a). SARPES data were taken under the mesh by looking for a region where the band map corresponded the most to the band map obtained in the reference spot.

The applied electric field does not introduce artifacts in SARPES. In order to exclude any unexpected measurement artifacts, we also tested the used setup with a system where no change of spin polarization is expected as a function of applied field. For this we measured the SARPES of 200-nm-thick films of $\mathrm{Bi}_{2} \mathrm{Se}_{3}$ grown on $\operatorname{InP}(111)$, because they can also be prepared by removing an amorphous Se cap under the Au mesh and possess a well-studied spinpolarized topological surface state $[20,21,33]$. Figures 8 (a) and 8(b) show the ARPES band map of the $\mathrm{Bi}_{2} \mathrm{Se}_{3}$ surface state in the reference spot and under the mesh, respectively, using the sample configuration of Fig. $1(\mathrm{~g})$. The influence of the mesh on the data quality is directly visible and even
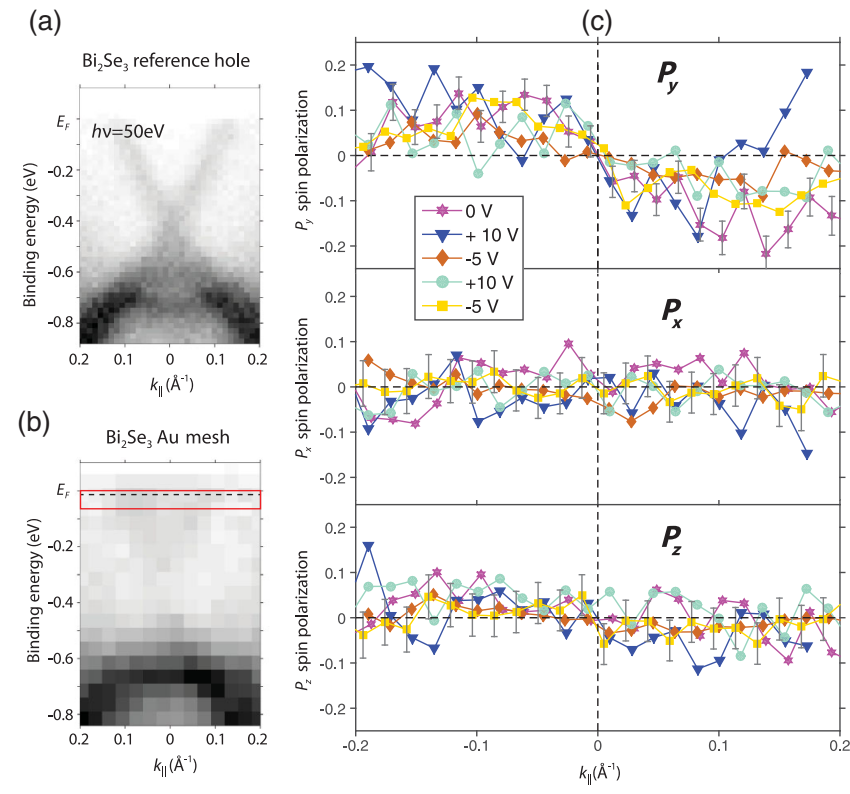

FIG. 8. (a) ARPES band map of a $\mathrm{Bi}_{2} \mathrm{Se}_{3}$ film measured in the reference hole with a photon energy of $50 \mathrm{eV}$ and $p$-polarized light. (b) Same as (a) but measured under the mesh. (c) Comparative operando SARPES data from $\mathrm{Bi}_{2} \mathrm{Se}_{3}$ measured under same conditions as $\alpha$-GeTe. The applied voltages are indicated by the symbols and colors. The red rectangle in (b) indicates the energy broadening and energy setting for the SARPES measurements. 


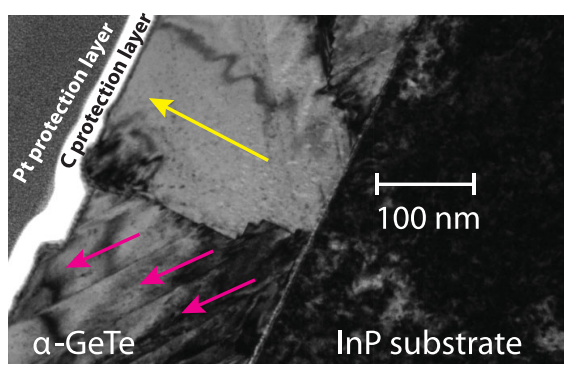

FIG. 9. TEM cross-section view of a $\alpha$-GeTe thin film with InP substrate distinguishing two types of grains, indicated by yellow and magenta arrows, respectively.

stronger as for GeTe in Fig. 2. In Fig. 8(c) the measured $P_{x, y, z}$ curves as a function of applied voltage are shown. The curves show no significant changes within the noise level; $P_{x}$ and $P_{z}$ remain zero and all $P_{y}$ data show the characteristic up-down shape going from negative to positive momenta.

The $\alpha$-GeTe films inspected by cross-section transmission electron microscopy in Fig. 9 revealed at least two characteristic grains in the epitaxial films. The yellow arrow indicates the main $\alpha$-GeTe(111) crystalline phase,

(a)

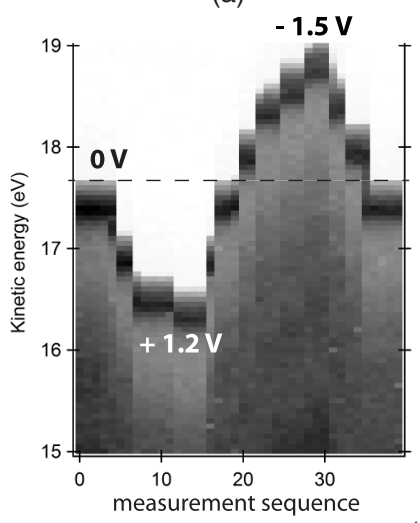

(c)
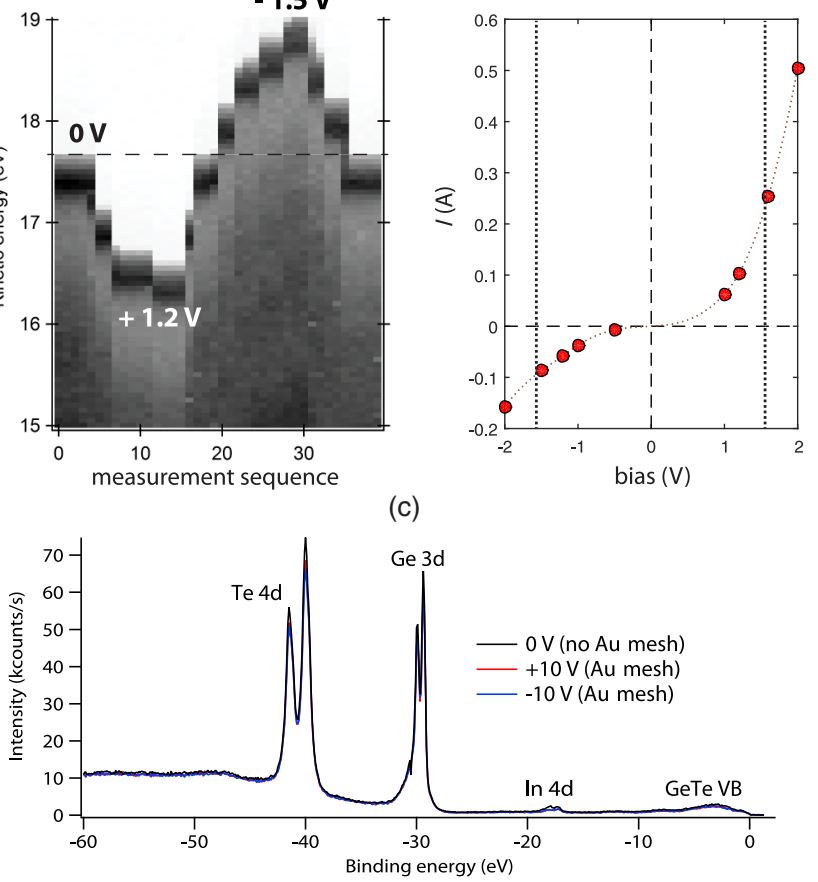

FIG. 10. (a) A series of bias-dependent shifts of the $\alpha$-GeTe valence band measured from the first gated sample depicted in Fig. 1(f); each measurement is an EDC measured for selected bias. (b) Typical semiconducting $I(V)$ curve measured on the first gated device (red dotted line is a guide to the eye). (c) XPS data measured from the second gated device at $h \nu=120 \mathrm{eV}$ in the reference spot seen in Fig. 7(c) and under the $\mathrm{Au}$ mesh for $\pm 10 \mathrm{~V}$. while the magenta arrows indicate laminar structures possibly related with the oblique domains illustrated in Fig. 1(d). We expect that the grain boundaries clearly visible in TEM favor the formation of ferroelastic domains. As the Au-mesh aperture is much larger compared to the characteristic length scale of these domains, and the beam spot is much larger than the mesh aperture, we do not expect that our operando SARPES is affected by domain wall motions.

The effect of the applied electric field on the $\alpha$-GeTe valence band in the first gated sample [Fig. 1(f)] is seen in Fig. 10(a). The corresponding $I(V)$ response shows a characteristic semiconductor behavior as seen in Fig. 10(b). Vertical dotted lines indicate the $\pm 1.6 \mathrm{~V}$ gate operating range. For the measurements the sample temperature was kept at $21 \pm 1 \mathrm{~K}$ with maximum heat dissipation of $\approx 300 \mathrm{~mW}$ at $+1.6 \mathrm{~V}$ bias. We note that for the second device with improved dielectric insulation [Fig. 1(g)], no current is measured with the used electronics $(<1 \mathrm{~mA}$ at $\pm 10 \mathrm{~V})$. Finally, Fig. 10(c) shows characteristic XPS spectra measured in the reference spot [Fig. 7(a), bottom] without bias, and under the Au mesh with $\pm 10 \mathrm{~V}$, indicating a stable $1: 1 \pm 3 \%$ GeTe stoichiometry.

Piezoelectric force microscopy measurements were performed at the NanoXAS end station at the SLS using a plain platinum tip at room temperature [34]. After SARPES measurements, the samples were transferred ex situ to the PFM chamber. The sample topography and PFM channels (amplitude and phase) are measured simultaneously. The PFM is measured close to tip resonance; no cross talk with topography was observed.

\section{APPENDIX B: SARPES VECTORIAL ANALYSIS}

By means of the two orthogonal Mott detectors of the COPHEE experimental station, it is possible to simultaneously measure the two in-plane and the out-of-plane spin-polarization directions, providing a three-dimensional system for measuring spin expectation values. Such a typical SARPES data set as presented in the main text consists of a total intensity spectrum $I_{t}$ and polarization spectra along the three spatial directions $P_{x, y, z}$ described in Fig. 2(d). From this data set, the spin-resolved spectra can be obtained by projecting the results on these spatial directions. The measured polarization and total intensity yield spin-resolved intensity spectra calculated as

$$
I_{x, y, z}^{\mathrm{up}}=\left(1+P_{x, y, z}\right) I_{t} / 2, I_{x, y, z}^{\mathrm{down}}=\left(1-P_{x, y, z}\right) I_{t} / 2 .
$$

In a well-established fitting routine the photoemission spectrum is first dissected into individual peaks and background and afterwards all polarization directions are fitted simultaneously to obtain spin vectors [35]. Figure 3 shows the total intensity $I_{t}$ of the MDC fitted with Voigt functions and a linear background indicated by magenta dashed line. 
Similarly, Fig. 4 shows the vectorial fitting of the EDC by Voigt functions and a background for a momentum near $0.1 \AA^{-1}$ along the $Z-U$ direction indicated in Fig. 2(e). We note that in order to asses the spin manipulations upon gate control in $\alpha$-GeTe, the full 3D spin analysis is helpful because the $\alpha$-GeTe Rashba splitting manifest a canted spin arrangement also in the in-plane spin texture [4]. The polarization curves in Fig. 3 (Fig. 4) are modeled until the best fit is reached by simultaneously fitting the MDC (EDC) intensity (purple dots) and the polarizations $P_{x}, P_{y}$, and $P_{z}$ (green lines).

Concerning the operando SARPES, we emphasize that during the $0 \rightarrow-1.6 \mathrm{~V}$ transition in Figs. 3(a) and 3(b), i.e., during the first poling of the as-grown $\alpha$-GeTe film, there is almost an ideal switching of all the spin polarization components, well observed also in the raw spin polarization curves $P_{x, y}$. These experimental observations are consistent with complete reversal of the spin vectors as depicted in Fig. 1(b).

\section{APPENDIX C: FIRST-PRINCIPLES CALCULATIONS}

To calculate structural properties associated with the ferroelectric switching in $\alpha$-GeTe, we perform density functional theory calculations employing the QUANTUM ESPRESSO package [36]. We used projector augmented wave pseudopotentials with kinetic energy cutoff of 40 Ry for wave functions and 240 Ry for charge density. For the exchange-correlation functional we used the generalized gradient approximation [37]. The surface properties were studied in a slab geometry. The $\alpha$-GeTe crystal along the [111] direction contains 18 atomic layers with hydrogen passivated Ge surface and physically relevant $\mathrm{Te}$ (111) surface. The Brillouin zone was sampled with $9 \times 9 k$ points. Atomic positions were relaxed using the quasiNewton algorithm based on the trust radius procedure.

[1] Concepts in Spin Electronics, edited by M. Maekawa (Oxford University Press, New York, 2006), pp. 43-90.

[2] D. Di Sante, P. Barone, R. Bertacco, and S. Picozzi, Electric Control of the Giant Rashba Effect in Bulk GeTe, Adv. Mater. 25, 509 (2013).

[3] S. Picozzi, Ferroelectric Rashba Semiconductors as a Novel Class of Multifunctional Materials, Front. Phys. 2, 10 (2014).

[4] J. Krempaský, H. Volfová, S. Muff, N. Pilet, G. Landolt, M. Radović, M. Shi, D. Kriegner, V. Holý, J. Braun, H. Ebert, F. Bisti, V. A. Rogalev, V. N. Strocov, G. Springholz, J. Minár, and J.H. Dil, Disentangling Bulk and Surface Rashba Effects in Ferroelectric $\alpha$-GeTe, Phys. Rev. B 94, 205111 (2016).

[5] J. Krempaský, S. Muff, F. Bisti, M. Fanciulli, H. Volfová, A. P. Weber, N. Pilet, P. Warnicke, F Bertran, H. Ebert, J. Braun, J. Minár, G. Springholz, J. H. Dil, and V. N. Strocov,
Entanglement and Manipulation of the Magnetic and SpinOrbit Order in Multiferroic $\mathrm{Ge}_{1-x} \mathrm{Mn}_{x} \mathrm{Te}$, Nat. Commun. 7, 13071 (2016).

[6] M. Liebmann et al., Giant Rashba-Type Spin Splitting in Ferroelectric GeTe(111), Adv. Mater. 28, 560 (2016).

[7] H. J. Elmers et al., Spin Mapping of Surface and Bulk Rashba States in Ferroelectric $\alpha$-GeTe(111) Films, Phys. Rev. B 94, 201403 (2016).

[8] G. S. Pawley, W. Cochran, R. A. Cowley, and G. Dolling, Diatomic Ferroelectrics, Phys. Rev. Lett. 17, 753 (1966).

[9] H. Przybylińska, G. Springholz, R. T. Lechner, M. Hassan, M. Wegscheider, W. Jantsch, and G. Bauer, Magnetic-FieldInduced Ferroelectric Polarization Reversal in the Multiferroic $\mathrm{Ge}_{1-x} \mathrm{Mn}_{x}$ Te Semiconductor, Phys. Rev. Lett. 112, 047202 (2014).

[10] D. Kriegner, J. Furthmüller, R. Kirchschlager, J. Endres, L. Horak, P. Cejpek, H. Reichlova, X. Marti, D. Primetzhofer, A. Ney, G. Bauer, F. Bechstedt, V. Holy, and G. Springholz, Ferroelectric Phase Transitions in Multiferroic $\mathrm{Ge}_{1-x} \mathrm{Mn}_{x}$ Te Driven by Local Lattice Distortions, Phys. Rev. B 94, 054112 (2016).

[11] J. Krempaský, M. Fanciulli, N. Pilet, J. Minár, W. Khan, M. Muntwiler, F. Bertran, S. Muff, A. P. Weber, V. N. Strocov, V. V. Volobuiev, G. Springholz, and J. H. Dil, Spin-Resolved Electronic Structure of Ferroelectric $\alpha$-GeTe and Multiferroic $\mathrm{Ge}_{1-x} \mathrm{Mn}_{x} \mathrm{Te}$, J. Phys. Chem. Solids (2017).

[12] W. Eerenstein, N. D. Mathur, and J. F. Scott, Multiferroic and Magnetoelectric Materials, Nature (London) 442, 759 (2006).

[13] M. Fiebig, Revival of the Magnetoelectric Effect, J. Phys. D 38, R123 (2005).

[14] A. V. Kolobov, D. J. Kim, A. Giussani, P. Fons, J. Tominaga, R. Calarco, and A. Gruverman, Ferroelectric Switching in Epitaxial GeTe Films, APL Mater. 2, 066101 (2014).

[15] J. E. Boschker, J. Momand, V. Bragaglia, R. Wang, K. Perumal, A. Giussani, B. J. Kooi, H. Riechert, and R. Calarco, Surface Reconstruction-Induced Coincidence Lattice Formation between Two-Dimensionally Bonded Materials and a Three-Dimensionally Bonded Substrate, Nano Lett. 14, 3534 (2014).

[16] R. Wang, J. E. Boschker, E. Bruyer, D. Di Sante, S. Picozzi, K. Perumal, A. Giussani, H. Riechert, and R. Calarco, Toward Truly Single Crystalline GeTe Films: The Relevance of the Substrate Surface, J. Phys. Chem. C 118, 29724 (2014).

[17] Fatigue in Ferroelectric Ceramics and Related Issues, edited by D. C. Lupascu (Springer, Berlin, 2004).

[18] J. H. Dil, Spin and Angle Resolved Photoemission on NonMagnetic Low-Dimensional Systems, J. Phys. Condens. Matter 21, 403001 (2009).

[19] T. Okuda and A. Kimura, Spin- and Angle-Resolved Photoemission of Strongly Spin-Orbit Coupled Systems, J. Phys. Soc. Jpn. 82, 021002 (2013).

[20] D. Hsieh, Y. Xia, L. Wray, D. Qian, A. Pal, J. H. Dil, J. Osterwalder, F. Meier, G. Bihlmayer, C. L. Kane, Y. S. Hor, R. J. Cava, and M. Z. Hasan, Observation of Unconventional Quantum Spin Textures in Topological Insulators, Science 323, 919 (2009).

[21] G. Landolt, S. Schreyeck, S. V. Eremeev, B. Slomski, S. Muff, J. Osterwalder, E. V. Chulkov, C. Gould, G. Karczewski, 
K. Brunner, H. Buhmann, L. W. Molenkamp, and J. H. Dil, Spin Texture of $\mathrm{Bi}_{2} \mathrm{Se}_{3}$ Thin Films in the Quantum Tunneling Limit, Phys. Rev. Lett. 112, 057601 (2014).

[22] A. H. Edwards, A. C. Pineda, P. A. Schultz, M. G. Martin, A. P. Thompson, H. P. Hjalmarson, and C. J. Umrigar, Electronic Structure of Intrinsic Defects in Crystalline Germanium Telluride, Phys. Rev. B 73, 045210 (2006).

[23] P. Zhang, P. Richard, T. Qian, Y.-M. Xu, X. Dai, and H. Ding, A Precise Method for Visualizing Dispersive Features in Image Plots, Rev. Sci. Instrum. 82, 043712 (2011).

[24] P. Höpfner, J. Schäfer, A. Fleszar, J. H. Dil, B. Slomski, F. Meier, C. Loho, C. Blumenstein, L. Patthey, W. Hanke, and R. Claessen, Three-Dimensional Spin Rotations at the Fermi Surface of a Strongly Spin-Orbit Coupled Surface System, Phys. Rev. Lett. 108, 186801 (2012).

[25] B. Slomski, G. Landolt, S. Muff, F. Meier, J. H. Dil, and J. Osterwalder, Interband Spin-Orbit Coupling between Anti-Parallel Spin States in Pb Quantum Well States, New J. Phys. 15, 125031 (2013).

[26] P. Gao, J. Britson, J. R. Jokisaari, C. T. Nelson, S.-H. Baek, Y. Wang, C.-B. Eom, L.-Q. Chen, and X. Pan, Atomic-Scale Mechanisms of Ferroelastic Domain-WallMediated Ferroelectric Switching, Nat. Commun. 4, 2791 (2013).

[27] K. Shportko, S. Kremers, M. Woda, D. Lencer, J. Robertson, and M. Wuttig, Resonant Bonding in Crystalline Phase-Change Materials, Nat. Mater. 7, 653 (2008).

[28] R. T. Lechner, G. Springholz, M. Hassan, H. Groiss, R. Kirchschlager, J. Stangl, N. Hrauda, and G. Bauer, Phase Separation and Exchange Biasing in the Ferromagnetic IV-VI Semiconductor $\mathrm{Ge}_{1-x} \mathrm{Mn}_{x} \mathrm{Te}$, Appl. Phys. Lett. 97, 023101 (2010).
[29] Y. A. Genenko, J. Glaum, M. J. Hoffmann, and K. Albe, Mechanisms of Aging and Fatigue in Ferroelectrics, Mater. Sci. Eng. B 192, 52 (2015).

[30] C. Verdier, D. C. Lupascu, and J. Rödel, Stability of Defects in Lead-Zirconate-Titanate after Unipolar Fatigue, Appl. Phys. Lett. 81, 2596 (2002).

[31] V. L. Deringer, M. Lumeij, and R. Dronskowski, Ab Initio Modeling of $\alpha$-GeTe(111) Surfaces, J. Phys. Chem. C 116, 15801 (2012).

[32] M. Hoesch, T. Greber, V. N. Petrov, M. Muntwiler, M. Hengsberger, W. Auwärter, and J. Osterwalder, SpinPolarized Fermi Surface Mapping, J. Electron Spectrosc. Relat. Phenom. 124, 263 (2002).

[33] J. Sánchez-Barriga, A. Varykhalov, G. Springholz, H. Steiner, R. Kirchschlager, G. Bauer, O. Caha, E. Schierle, E. Weschke, A. A. Ünal, S. Valencia, M. Dunst, J. Braun, H. Ebert, J. Minár, E. Golias, L. V. Yashina, A. Ney, V. Holý, and O. Rader, Nonmagnetic Band Gap at the Dirac Point of the Magnetic Topological Insulator $\left(\mathrm{Bi}_{1-x} \mathrm{Mn}_{x}\right)_{2} \mathrm{Se}_{3}$, Nat. Commun. 7, 10559 (2016).

[34] N. Pilet, J. Raabe, S. E. Stevenson, S. Romer, L. Bernard, C. R. McNeill, R. H. Fink, H. J. Hug, and C. Quitmann, Nanostructure Characterization by a Combined X-Ray Absorption/Scanning Force Microscopy System, Nanotechnology 23, 475708 (2012).

[35] F. Meier, J. H. Dil, and J. Osterwalder, Measuring Spin Polarization Vectors in Angle-Resolved Photoemission Spectroscopy, New J. Phys. 11, 125008 (2009).

[36] P. Giannozzi et al., QUANTUM ESPRESSO: A Modular and Open-Source Software Project for Quantum Simulations of Materials, J. Phys. Condens. Matter 21, 395502 (2009).

[37] J. P. Perdew, K. Burke, and M. Ernzerhof, Generalized Gradient Approximation Made Simple, Phys. Rev. Lett. 77, 3865 (1996). 